Article

\title{
From Graphite to Laccase Biofunctionalized Few-Layer Graphene: A "One Pot" Approach Using a Chimeric Enzyme
}

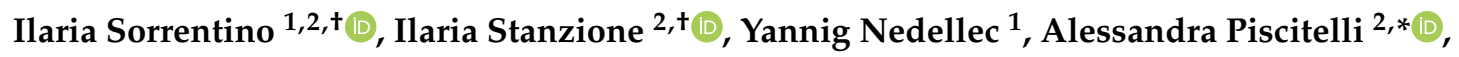 \\ Paola Giardina ${ }^{2}(1)$ and Alan Le Goff ${ }^{1, *}$ \\ 1 Department of Molecular Chemistry, University Grenoble Alpes, CNRS, 38000 Grenoble, France; \\ ilaria.sorrentino@univ-grenoble-alpes.fr (I.S.); yannig.nedellec@univ-grenoble-alpes.fr (Y.N.) \\ 2 Department of Chemical Sciences, University Federico II, 80126 Naples, Italy; \\ ilaria.stanzione@unina.it (I.S.); giardina@unina.it (P.G.) \\ * Correspondence: apiscite@unina.it (A.P.); alan.le-goff@univ-grenoble-alpes.fr (A.L.G.) \\ + These authors contributed equally to the work.
}

Received: 28 April 2020; Accepted: 22 May 2020; Published: 26 May 2020

\begin{abstract}
A chimeric enzyme based on the genetic fusion of a laccase with a hydrophobin domain was employed to functionalize few-layer graphene, previously exfoliated from graphite in the presence of the hydrophobin. The as-produced, biofunctionalized few-layer graphene was characterized by electrochemistry and Raman spectroscopy, and finally employed in the biosensing of phenols such as catechol and dopamine. This strategy paves the way for the functionalization of nanomaterials by hydrophobin domains of chimeric enzymes and their use in a variety of electrochemical applications.
\end{abstract}

Keywords: laccase; hydrophobin; graphene

\section{Introduction}

Graphene is a two-dimensional sheet of $\mathrm{sp}^{2}$-hybridized carbon that exhibits unparalleled properties such as high planar surface, superlative mechanical strength, and remarkable thermal and electrical conductivity. Due to its extraordinary structure and fascinating properties, graphene is the most studied nanomaterial and can be integrated as the core of cutting-edge devices in many types of applications, ranging from microelectronics to electrochemical energy harvesting systems [1-8]. In biosensing in particular, graphene acts as a conductive platform for biomolecule immobilization and electrochemical detection of bioanalytes [9-14]. Graphene-based electrochemical biosensing has relied on the recent developments in the study of graphene electrochemical properties, its production, and biofunctionalization. Different techniques have been investigated for the production of graphene such as scotch-tape transfer, chemical vapor deposition (CVD) growth, and chemically or electrochemically reduced graphene oxide. These strategies lead to different nanomaterials in terms of size, edge and basal defects, number of layers, and oxygenated defect content. Although CVD produces a large surface of monolayer graphene, soft exfoliation of graphite has also been able to provide low-cost access to few-layer graphene (FLG) dispersions. The dispersion stability is one of the main challenges to address during the exfoliation procedure, as in general, the re/aggregation of exfoliated material is minimized by using organic solvents or surfactant-water solutions [15-17]. Like other nanomaterials, graphene is a very suitable platform for enzyme immobilization thanks to its high surface area, dispersion in solution, and tunable surface chemistry. Intense efforts have been devoted to this research field in the last five years, resulting in the immobilization of different enzymes for various applications [18]. However, the hydrophobic interactions driving the direct immobilization of active proteins on graphene surface are often difficult to achieve, and modifications of the protein 
3D structures can occur with detrimental effects on their functionality [19]. Two main approaches have been implemented, and often combined, to overcome this issue, such as the use of graphene oxide (GO) whose surface is more hydrophilic, or the exploitation of graphene-based composites, such as microcellulose, chitosan, and various metal oxides [18].

Recently, proteins prone to forming amyloid structures have been proven to be able to biofunctionalize graphene. In this respect, the fungal self-assembling class I hydrophobin Vmh2 from Pleurotus ostreatus has been successfully exploited to disperse and stabilize FLG in ethanol-water mixtures by ultrasonic wave exfoliation. [20] Hydrophobins (HFB) are a family of small self-assembling proteins produced by filamentous fungi, and can be divided into two classes that differ for the nature of the amphipathic layers that they form. Fibrillar structures formed by class I HFB are extremely robust, are disassembled only in strong acids, and share structural properties with amyloid fibrils [21]. HFB efficiently adheres to several hydrophobic surfaces, including 2D materials, such as graphene [20]. This ability has been further exploited by genetic fusion of the hydrophobin to biotechnologically relevant proteins that can be immobilized on various surfaces, obtaining the so-called "self-immobilizing" proteins/enzymes [22]. Recently a new chimeric protein was designed to combine the HFB Vmh2 with a laccase enzyme (Lac), Lac-Vmh2 [23]. Laccases (p-diphenol-dioxygenoxidoreductases; EC 1.10.3.2) are multicopper oxidases able to catalyze the oxidation of a wide range of aromatic substrates using oxygen as co-substrate and producing water as the only by-product. These enzymes are promising biocatalysts with possible applications in bioremediation, chemical synthesis, biobleaching of paper pulp, and biosensing [24]. Laccases have been immobilized on various carriers, using different methods with both advantages and drawbacks. Among the various enzymes, the laccase POXA1b from P. ostreatus was chosen for its peculiar characteristics such as its stability and activity in a wide range of $\mathrm{pHs}$ and temperatures, as well as its high redox potential [25]. The produced Lac-Vmh2 allowed achievement of simple and stable immobilization of the enzyme on polystyrene [23].

The main purpose of this work was to advance the biofunctionalization of graphene with Vmh2, immobilizing laccase on FLG by using the fusion protein Lac-Vmh2 through a "one-pot" approach. The presented method is easy, eco-friendly, and versatile, because, in principle, a wide variety of different HFB chimera proteins can be used in this one-pot exfoliation/functionalization procedure. As a proof of concept, the as-prepared Lac-Vmh2/FLG was used for the modification of Glassy Carbon (GC) electrodes to build an electrochemical sensor for phenolic compounds, such as catechol, a well-known environmental pollutant, and dopamine, a renowned neurotransmitter.

\section{Results and Discussion}

\subsection{Laccase Immobilization on FLG}

Graphite exfoliation was carried out, as previously described, by exposing mixtures of Vmh2 protein and graphite to ultrasonic waves [20]. Vmh2-exfoliated graphene is generally stable in $60 \%$ ethanol (EtOH) thanks to the presence of the HFB. On the other hand, enzymes are usually used and stable in aqueous buffers. Thus, conditions have to be assessed to preserve both the stability of graphene dispersion and the enzyme activity. The wild-type PoxA1b laccase and Lac-Vmh2 were dialyzed toward different ethanol concentration $(20 \%, 40 \%, 50 \%$ and $60 \% \mathrm{EtOH}$,) and $10 \mathrm{mM}$ Tris- $\mathrm{HCl}$ $\mathrm{pH} 8$, in order to test the stability of the enzyme in these conditions. Concomitantly, the Vmh2 graphene samples in $60 \% \mathrm{EtOH}$ were centrifuged and pellets were solubilized with or without the addition of $0.05 \mathrm{mg} \mathrm{mL}^{-1}$ of Vmh2 in the same conditions tested for enzymatic stability. The optimal condition for the graphene stability was 50\% $\mathrm{EtOH}+\mathrm{Vmh} 2$ and $40 \% \mathrm{EtOH}+\mathrm{Vmh} 2$ (Figure S1). On the other hand, the enzyme stability was reasonable up to $40 \% \mathrm{EtOH}$ (Table S1). Thus, looking both at the enzyme and the graphene stability, the $40 \% \mathrm{EtOH}$ was selected as the optimal solvent for graphite exfoliation in the presence of laccase. 
To study and optimize the condition of laccase immobilization on the FLG, several tests were performed (Figure 1). Addition of the enzymes (wild-type or chimera) was performed after exfoliation of graphite in the presence of $\mathrm{Vmh} 2$ (route A). Addition of the enzyme solutions to graphite powder was performed at the beginning of the exfoliation (route B). Or, in situ exfoliation of graphite with Vmh2 was followed by the addition of the enzymes in the last $10 \mathrm{~min}$ of sonication (route C). Each route was compared by measuring the enzymatic activity [25] of the biofunctionalized FLG (previously separated after centrifugation at 13,000 rpm for $15 \mathrm{~min}$ ) obtained after centrifugation. Results are displayed in Table S2. Route A led to final no enzymatic activity, indicating that negligible amounts of enzymes can be immobilized on FLG after exfoliation of graphite with Vmh2. When the chimera was used to exfoliate graphene from graphite (route B), the extensive ultrasonication time led to a complete loss of the enzymatic activity of laccase. To avoid a long exposure of the enzymes to ultrasonic waves, route $\mathrm{C}$ was used in the course of this work. In the latter route, wild-type or chimera were added during the last $10 \mathrm{~min}$ of the exfoliation process in order to prevent enzyme inactivation. An immobilization yield of $5 \%$ and $11 \%$, for PoxA1b or Lac-Vmh2, respectively, was estimated, considering the enzymatic activity before and after immobilization. According to the activity of the immobilized Lac-Vmh2, this corresponded to an enzyme loading of about $0.4 \mathrm{U}$ per mg of FLG (Table 1). Lac-Vmh2 showed a slight increased amount of attached enzyme as compared to the wild-type enzyme. Indeed, POXA1b was able to stick to graphene, as already observed using polystyrene [23]. Nevertheless, the stability of the biofunctionalized graphene obtained with Lac-Vmh2 was higher than that of the wild-type enzyme, in terms of both activity and adhesion (Table 1).

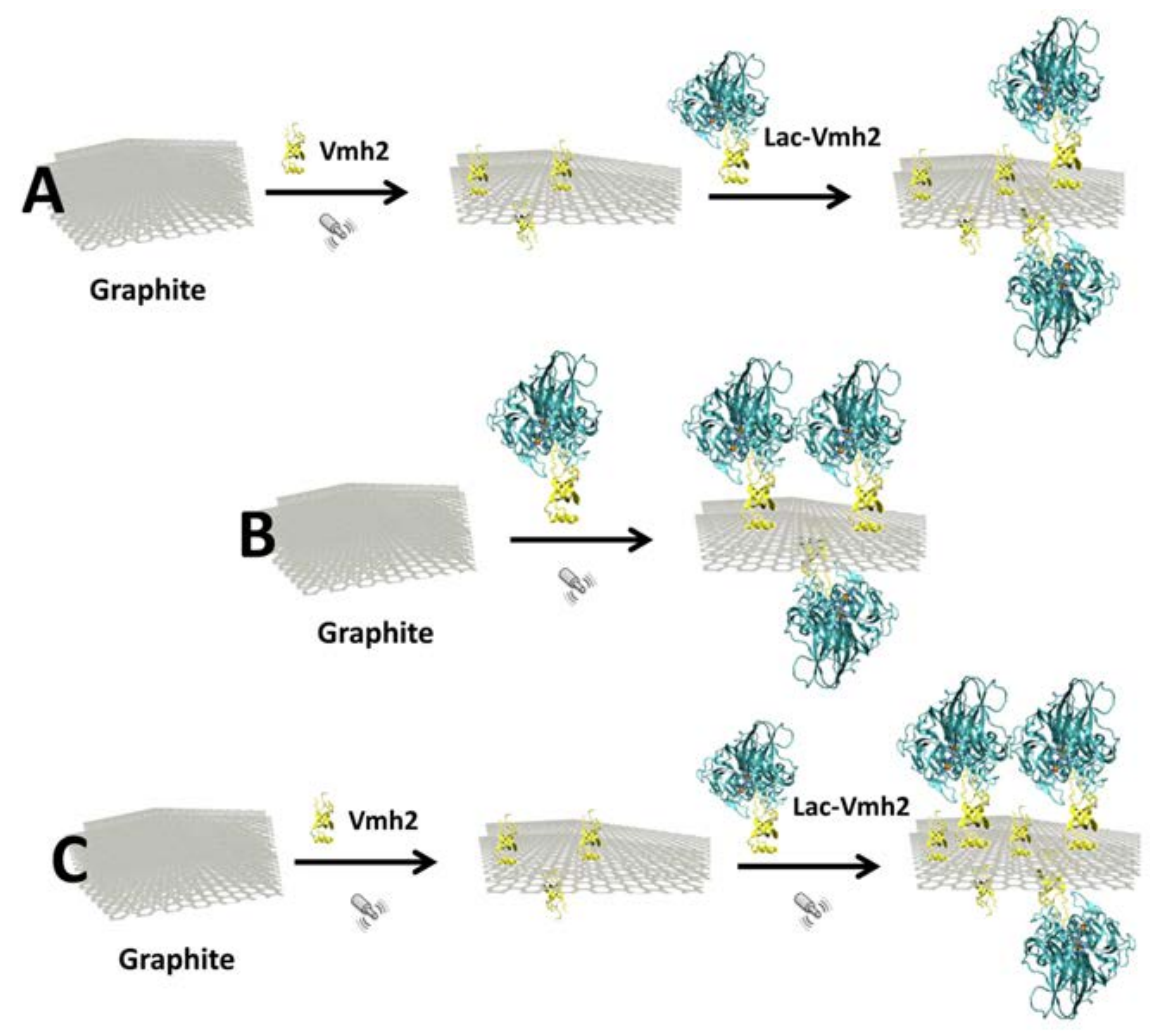

Figure 1. Tests performed to exfoliate and biofunctionalize graphite with laccase chimera, Lac-Vmh2. (A) graphite exfoliation in the presence of $\operatorname{Vmh} 2(\sim 5 \mathrm{~h})$ and subsequent addition of Lac-Vmh2; (B) graphite exfoliation in the presence of both Vmh2 and Lac-Vmh2 ( $5 \mathrm{~h})$; (C) graphite exfoliation in the presence of $\operatorname{Vmh} 2(\sim 5 \mathrm{~h})$ and addition of Lac-Vmh2 in the last $10 \mathrm{~min}$ of exfoliation. 
Table 1. Summary of the sample enzymatic activity and their characteristics.

\begin{tabular}{cccc}
\hline Samples & $\begin{array}{c}\text { Units in Milligrams of } \\
\text { Graphene (U } \mathbf{~ m - 1 )}\end{array}$ & $\mathbf{t}_{\mathbf{1 / 2}}$ (Days) & Activity after Washing \\
\hline Graphene/PoxA1b & $0.3 \pm 0.1$ & 17 & Stable up to the second wash \\
Graphene/Lac-Vmh2 & $0.4 \pm 0.1$ & 26 & Stable up to the fourth wash \\
\hline
\end{tabular}

The biofunctionalized graphene was stable at least for 30 days (Figure $2 \mathrm{~A}$ ).
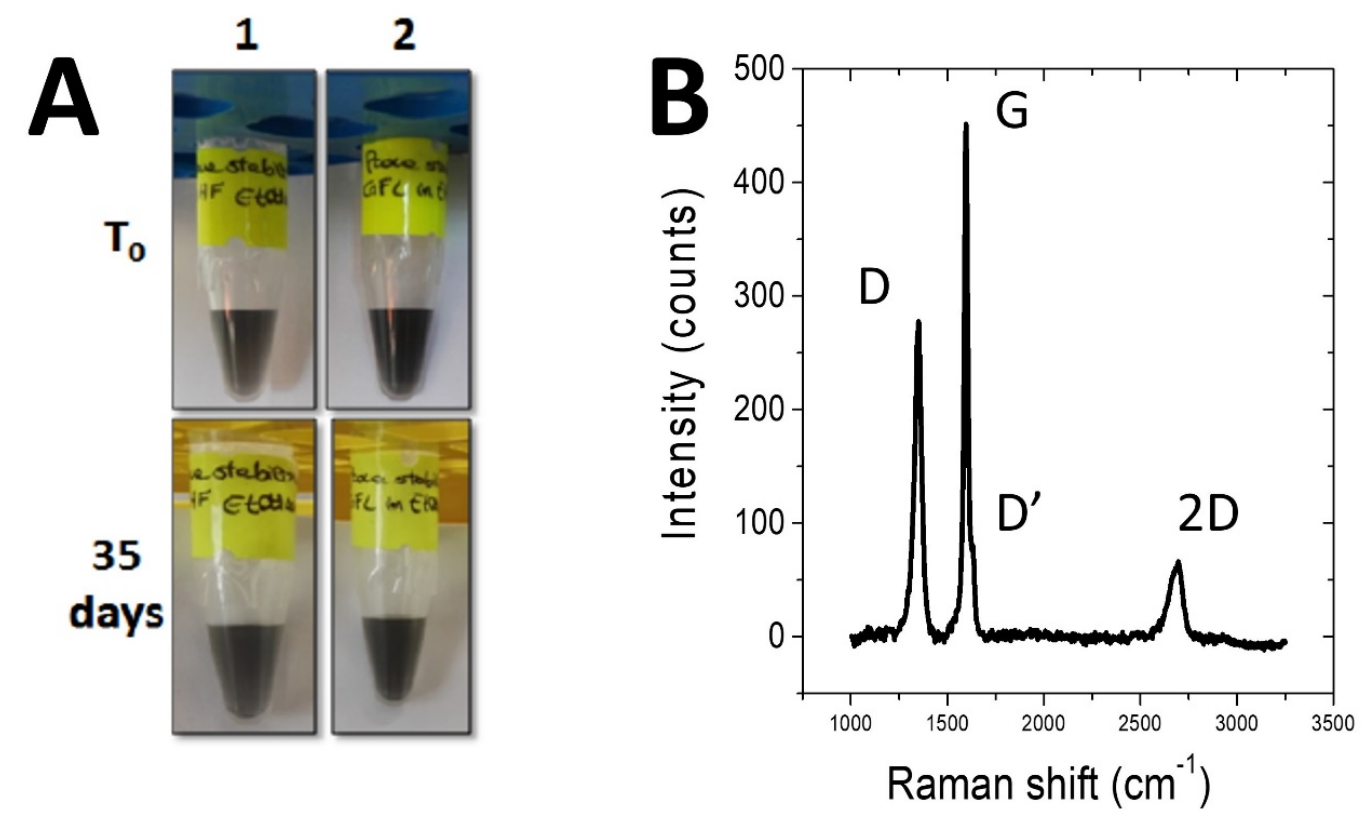

Figure 2. (A) Stability of graphene dispersion at time zero and after 35 days: lane1: Graphene/PoxA1b; lane 2: Graphene/Lac-Vmh2. (B) Representative Raman spectrum of a Lac-Vmh2-biofunctionlized few-layer graphene (FLG) film.

Raman spectroscopy was also performed in order to characterize the number of graphene layers for these biofunctionalized FLG (Figure 2B). According to Ferrari's work [26], the high-energy band observed at $2700 \mathrm{~cm}^{-1}$ stems for the presence of only few graphene layers $(<5$ layers), as already observed in the case of the native Vmh2 hydrophobin [20]. It is noteworthy that the Raman spectrum was also performed after several months on the same stock solution of Lac-Vmh2-functionalized FLG without showing any restacking nor aggregation phenomena.

\subsection{Exploitation of Biofunctionalized FLG in Electrochemical Biosensing}

GC electrodes were then modified by drop-casting a solution of biofunctionalized FLG. The incubation was performed at room temperature until the electrode was completely dry, and then several washes with citrate-phosphate buffer $\mathrm{pH} 5.0$ were executed to eliminate the unbound sample.

Figure 3 displays a typical SEM image of biofunctionalized FLG deposited onto planar gold electrode. This underlines the homogenous dispersion of FLG both in solution and at the surface of the electrode. According to size distribution study, the sizes of biofunctionalized FLG are mostly below $2 \mu \mathrm{m}^{2}$. These nanostructured bioelectrodes were employed for biosensing experiments. The principle of laccase biosensors is based on the enzymatic oxidation of phenols or o-diphenols into quinones, the latter being subsequently reduced at the nanostructured electrode polarized at a redox potential required for the electroreduction of quinone into phenols, that is, $\mathrm{E}=-0.2 \mathrm{~V}$ vs. saturated calomel electrode (SCE). The regeneration of the catechol derivative triggers an amplification cycle of "enzymatic oxidation/electrochemical reduction", which increases biosensing sensitivity. 


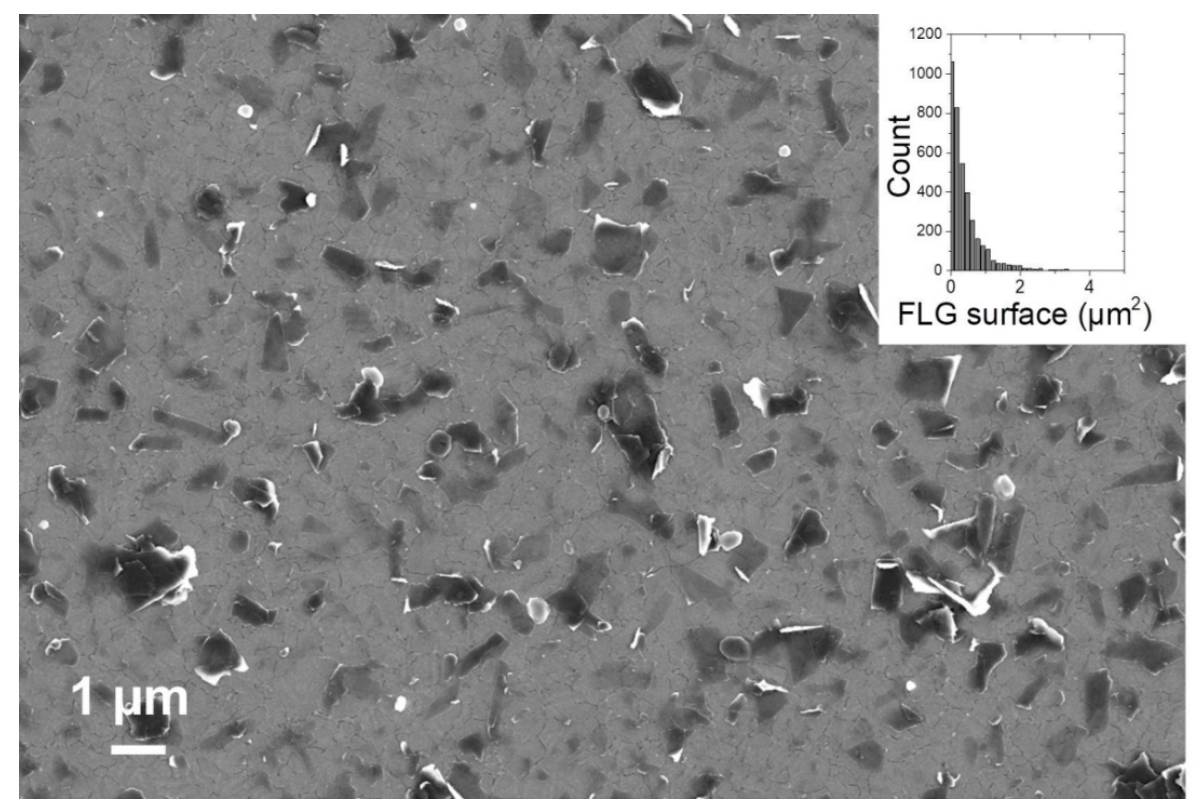

Figure 3. SEM image of biofunctionalized FLG at the surface of a gold electrode; (inset) size distribution of biofunctionalized FLG obtained by Image.

Addition of both catechol and dopamine was monitored at Lac-Vmh2-biofunctionalized FLG electrodes. Figure $4 \mathrm{~A}$ displays a representative chronoamperometry experiment performed upon the addition of catechol.
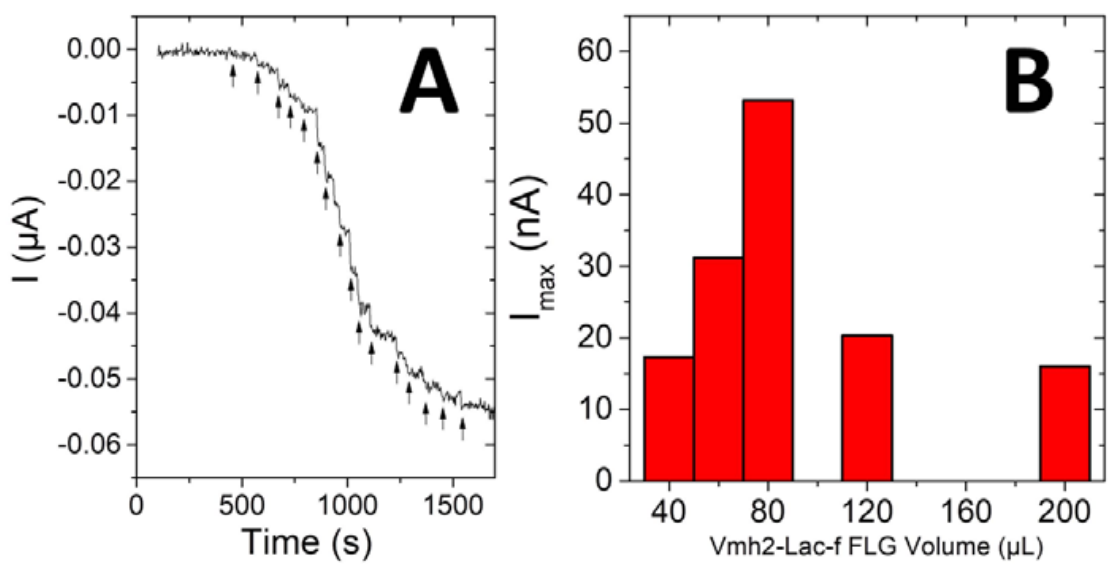

Figure 4. (A) Chronoamperometry performed at Lac-Vmh2-biofunctionlized FLG electrode after successive additions of catechol (indicated by the arrows, applied potential $=-0.2 \mathrm{~V}$ vs. saturated calomel electrode (SCE), $0.1 \mathrm{M}$ Phosphate-buffered saline (PBS), $\mathrm{pH} 6,25^{\circ} \mathrm{C}$ ). (B) Plot of the maximum current towards volume of drop-coated Lac-Vmh2-biofunctionlized FLG solutions.

Chronoamperometry at different volumes of FLG (Figure 4B) were performed, showing that an optimum value of biofunctionalized FLG was reached after the drop-coating of $80 \mu \mathrm{L}$ (16 mU) solution on GC electrodes. These experiments underlined the fact that an optimal volume of FLG was needed in order to maximize the amount of immobilized enzymes while also providing efficient diffusion of catechol into the nanostructured FLG conductive film. In order to indirectly confirm the interaction between the Vmh2 domain of the chimera and graphene layer, the wild-type POXA1b was also used in the exfoliation process of FLG. However, on the contrary to the Lac-Vmh2-biofunctionlized FLG electrode (Figure 4A), negligible current was observed for catechol oxidation, underlining the fact that less adsorption of POXA1b was observed on FLG. This also demonstrated the important role of the Vmh2 domain in order to immobilize laccase at the surface of FLG. 
Figure 5 displays the calibration curves for catechol and dopamine recorded at these electrodes. The shape of the curves was governed by the enzymatic reaction that was reliability modelized according to typical Michaelis-Menten kinetics. Table 2 summarizes the electrochemical characteristics of the modelized curves.

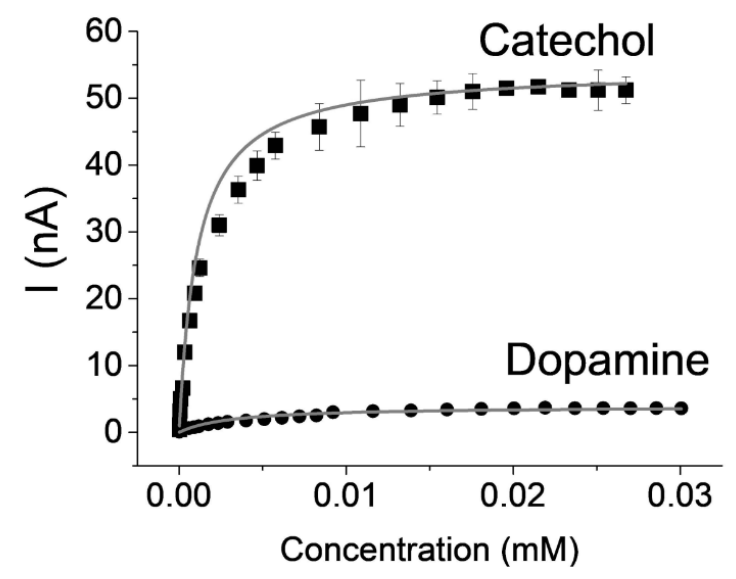

Figure 5. Plot of the catalytic current towards increasing concentrations of $(\boldsymbol{\square})$ catechol and $(\bullet)$ dopamine for electrodes (measurements performed by chronoamperometry at $\mathrm{E}=-0.2 \mathrm{~V}$ vs. SCE, $0.1 \mathrm{M}$ PBS, $\left.\mathrm{pH} 6,25^{\circ} \mathrm{C}\right)$.

Table 2. Electrochemical characteristics obtained after modelization of the curve according Michaelis-Menten kinetics; equation: $\mathrm{I}=\left(\mathrm{I}_{\mathrm{MAX}} \times[\right.$ substrate $\left.]\right) /\left(\mathrm{K}_{\mathrm{M}}+[\right.$ substrate $\left.]\right)$.

\begin{tabular}{cccc}
\hline Substrate & $\mathbf{K}_{\mathbf{M}}(\mathbf{m M})$ & $\mathbf{I}_{\mathbf{M A X}}\left(\mathbf{n A ~ c m} \mathbf{~ c m}^{-\mathbf{2}}\right)$ & $\boldsymbol{R}^{\mathbf{2}}$ \\
\hline Catechol & 1.1 & 775.7 & 0.99 \\
Dopamine & 3.0 & 55.5 & 0.95 \\
\hline
\end{tabular}

The apparent Michaelis-Menten constant $\left(\mathrm{K}_{\mathrm{Mapp}}\right)$ reflected the enzyme-substrate affinity, which was higher in the case of dopamine when compared to catechol for laccase. A lower Km value is advantageous in the case of laccase-based sensors in order to observe linear range with a minimal limit of detection and a maximal sensitivity. Laccase has a better affinity for catechol compared to dopamine. This is the reason why biosensors based on laccases always exhibit better performance towards catechol as compared to dopamine.

The linear part of the curve is shown in Figure 6.
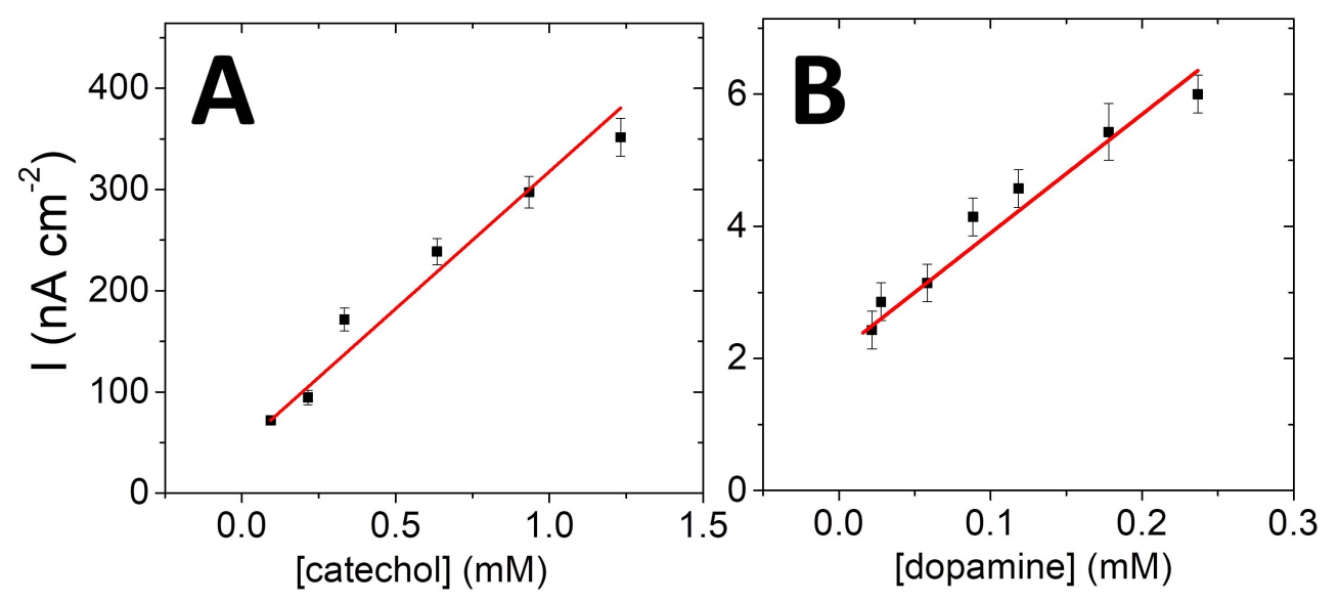

Figure 6. Plot of the linear part of the catalytic current density towards concentration of $(\mathrm{A})$ catechol and $(\mathbf{B})$ dopamine $\left(\mathrm{E}_{\text {applied }}=-0.2 \mathrm{~V}\right.$ vs. SCE, $\left.0.1 \mathrm{M} \mathrm{PBS}, \mathrm{pH} 6,25^{\circ} \mathrm{C}\right)$. 
Limit of detection (LOD) of $20 \mu \mathrm{M}$ was separately measured for both catechol and dopamine, with sensitivity of $0.27 \mathrm{~mA} \mathrm{M}{ }^{-1} \mathrm{~cm}^{-2}\left(R^{2}=0.97\right)$ towards catechol and $16.4 \mu \mathrm{A} \mathrm{M}^{-1} \mathrm{~cm}^{-2}\left(R^{2}=0.96\right)$ towards dopamine being measured with respective linear ranges of 20 to $1000 \mu \mathrm{M}$ and 20 to $250 \mu \mathrm{M}$. Although the most efficient phenolic biosensors are based on a combination of tyrosinases and laccases, $[27,28]$ or based on the use of redox hydrogels $[29,30]$, this type of nanostructured biosensor approaches the performances of other types of biosensors that associate graphene and laccases [29,31,32].

\section{Materials and Methods}

All products were purchased from Sigma-Aldrich and were used without further purification. All solvents were of analytical grade. Distilled water was passed through a Milli-Q water purification system to obtain ultrapure water at $18.2 \mathrm{M} \Omega \mathrm{cm}^{-1}$. Phosphate-buffered saline (PBS) solution was prepared from Milli-Q water.

\subsection{Electrochemical Measurements}

The electrochemical experiments were carried out in a three-electrode electrochemical cell using Ametek Multipotentiostat Princeton Applied Research (Wokingham, UK). A Pt wire was used as the counter electrode and the saturated calomel electrode (SCE) served as the reference electrode. All experiments were conducted at room temperature. All simulated curves were obtained via Origin Pro 9.0. Error bars were estimated from three measurements recorded per sample.

\subsection{Laccase Enzymes}

Both fusion proteins Lac-Vmh2 and wild-type enzyme POXA1b were produced and secreted by the yeast Pichia pastoris in the culture media. The supernatant, after centrifugation for $15 \mathrm{~min}$ at $6000 \mathrm{rpm}$ at $4{ }^{\circ} \mathrm{C}$, was concentrated and dialyzed towards $50 \mathrm{mM}$ Tris- $\mathrm{HCl}$ buffer, $\mathrm{pH}$ 8.0, using Centricon Centrifugal Filter Units 10kDa (Merck, Darmstadt, Germany). The laccase enzymes are used without additional purification steps. The total protein concentration was determined using the Pierce 660 method (Thermo Fischer Scientific, Waltham, Massachusetts, MA, USA) and using (Bovine Serum Albumin) BSA as the standard. The laccase activity was assayed at room temperature, monitoring the oxidation of ABTS (2,2'-azino-bis(3-ethylbenzothiazoline-6-sulphonic acid)) at $420 \mathrm{~nm}$ $\left(\varepsilon_{420 \mathrm{~nm}}=3.6 \times 10^{4} \mathrm{M}^{-1} \mathrm{~cm}^{-1}\right)$ 一the assay mixture contained $2 \mathrm{mM} \mathrm{ABTS}$ and $0.1 \mathrm{M}$ sodium citrate buffer, $\mathrm{pH} 3.0$.

\subsection{In Situ Exfoliation of Graphite}

Graphite powder, $1 \mathrm{mg} / \mathrm{mL}$ (Aldrich, 332461, mesh number of grains $+100,>75 \%$ ), was exfoliated in batches of $7.5 \mathrm{~mL}$ of $60 \% \mathrm{v} / \mathrm{v}$ ethanol (EtOH) in MilliQ water (in $20 \mathrm{~mL}$ flasks) and $7.5 \mathrm{~mL}$ of $50 \mu \mathrm{g} / \mathrm{mL}$ Vmh2, using a medium power tip sonicator (Ultrasound SONOPLUS HD3200, maximum power $200 \mathrm{~W}$, working frequency $20 \mathrm{kHz}$, KE-76 probe, running at 15\% amplitude. BANDELIN, Berlin Germany) and cooling the system in an ice bath. The exfoliation was stopped when the energy value was $450 \mathrm{KJ}$ (about $5 \mathrm{~h}$ of sonication time). To remove the unexfoliated material, consecutive $40 \mathrm{~min}$ centrifugations at increased centrifugal force were performed $(40 \times, 160 \times$ and $620 \times g)$. We characterized and used the dispersions obtained after the last centrifugation.

Three types of experiments were tested with the laccase enzymes:

- A $7 \mathrm{~mL}$ solution of PoxA1b or Lac-Vmh2 in 40\% EtOH was added to Vmh2-exfoliated graphene and incubated at $4{ }^{\circ} \mathrm{C}$ whilst stirring continuously.

- The immobilization was performed by adding the wild-type or chimeric enzyme solution to graphite powder at the beginning of the exfoliation.

- The wild-type or chimeric enzyme solution was added during the last $10 \mathrm{~min}$ of exfoliation. Indeed, the inactivation of the enzyme when higher sonication time was used has been previously 
verified. The process was performed normalizing the activity units ( $4 \mathrm{U}_{\text {tot }}$ for both) between wild-type POXA1b and Lac-Vmh2 (0.16 $\mathrm{mg}$ and $0.44 \mathrm{mg}$, respectively).

\section{Conclusions}

This work shows that Lac-Vmh2 chimera enzyme can be used both as a surfactant of FLG while also providing enzymatic activity to biofunctionalized nanomaterials. This original biofunctionalization technique represents a soft and biocompatible technique compared to the use of more classic long-alkyl-chain surfactants, leading to stable exfoliation of FLGs. The possibility to merge Vmh2 with active enzymes into chimera enzymes brings novel physico-chemical properties to this exfoliation technique. Here, deposition of these biofunctionalized FLGs on electrodes affords the fabrication of catechol and dopamine biosensors. This novel strategy of functionalizing carbon nanomaterials with specific chimeric enzymes paves the way for the development of many types of novel chimeric enzymes that can be developed for a variety of applications involving multienzymatic systems and biofunctionalization of nanomaterials.

Supplementary Materials: Supplementary materials can be found at http:/www.mdpi.com/1422-0067/21/11/ 3741/s1. Figure S1: Stability of graphene dispersion in different solvents; Table S1: $t_{1 / 2}$ of laccases in different buffers; Table S2: Summary of the immobilization experiments.

Author Contributions: Experiments, I.S. (Ilaria Stanzione) and I.S. (Ilaria Sorrentino); Raman spectroscopy, Y.N.; writing-review and editing, A.L.G., A.P., and P.G.; supervision, A.L.G., A.P., and P.G.; project administration, A.L.G., A.P., and P.G.; funding acquisition, A.L.G., A.P., and P.G.; I.S. (Ilaria Stanzione) and I.S. (Ilaria Sorrentino) contributed equally to this work. All authors have read and agreed to the published version of the manuscript.

Funding: This project is funded by the Italian Education, University and Research Ministry (MIUR) and the French National Research Agency (ANR), and co-funded by European Union's Horizon 2020 research and innovation program under the framework of ERA-NET Cofund MarTERA (Maritime and Marine Technologies for a new Era), FLAshMoB, Functional Amyloid chimera for Marine Biosensing (ID: 172). This work was also supported by the Agence Nationale de la Recherche through the LabEx ARCANE programme (ANR-11-LABX-0003-01) and the Graduate School on Chemistry, Biology and Health of Univ Grenoble Alpes CBH-EUR-GS (ANR-17-EURE-0003). The authors acknowledge support from the plateforme de Chimie NanoBio ICMG FR 2607 (PCN-ICMG).

Conflicts of Interest: The authors declare no conflict of interest.

\section{References}

1. Pumera, M. Voltammetry of carbon nanotubes and graphenes: Excitement, disappointment, and reality. Chem. Rec. 2012, 12, 201-213. [CrossRef] [PubMed]

2. Brownson, D.A.C.; Lacombe, A.C.; Gómez-Mingot, M.; Banks, C.E. Graphene oxide gives rise to unique and intriguing voltammetry. Rsc. Adv. 2011, 2, 665-668. [CrossRef]

3. Brownson, D.A.C.; Munro, L.J.; Kampouris, D.K.; Banks, C.E. Electrochemistry of graphene: Not such a beneficial electrode material? Rsc. Adv. 2011, 1, 978-988. [CrossRef]

4. Kampouris, D.K.; Banks, C.E. Exploring the physicoelectrochemical properties of graphene. Chem. Commun. 2010, 46, 8986-8988. [CrossRef] [PubMed]

5. Novoselov, K.S.; Fal'ko, V.I.; Colombo, L.; Gellert, P.R.; Schwab, M.G.; Kim, K. A roadmap for graphene. Nature 2012, 490, 192-200. [CrossRef]

6. Ferrari, A.C.; Bonaccorso, F.; Fal'ko, V.; Novoselov, K.S.; Roche, S.; Bøggild, P.; Borini, S.; Koppens, F.H.L.; Palermo, V.; Pugno, N.; et al. Science and technology roadmap for graphene, related two-dimensional crystals, and hybrid systems. Nanoscale 2015, 7, 4598-4810. [CrossRef]

7. Zhang, F.; Li, Y.-H.; Li, J.-Y.; Tang, Z.-R.; Xu, Y.-J. 3D graphene-based gel photocatalysts for environmental pollutants degradation. Environ. Pollut. 2019, 253, 365-376. [CrossRef]

8. Zhang, N.; Yang, M.-Q.; Liu, S.; Sun, Y.; Xu, Y.-J. Waltzing with the Versatile Platform of Graphene to Synthesize Composite Photocatalysts. Chem. Rev. 2015, 115, 10307-10377. [CrossRef]

9. Fritea, L.; Tertis, M.; Sandulescu, R.; Cristea, C. Enzyme-Graphene Platforms for Electrochemical Biosensor Design with Biomedical Applications. In Methods in Enzymology; Kumar, C.V., Ed.; Academic Press: Cambridge, MA, USA, 2018; Chapter Eleven; Volume 609, pp. 293-333.

10. Pumera, M. Graphene in biosensing. Mater. Today 2011, 14, 308-315. [CrossRef] 
11. Zhang, W.; Jia, B.; Furumai, H. Fabrication of graphene film composite electrochemical biosensor as a pre-screening algal toxin detection tool in the event of water contamination. Sci. Rep. 2018, 8, 1-10. [CrossRef]

12. Lalaoui, N.; Le Goff, A.; Holzinger, M.; Mermoux, M.; Cosnier, S. Wiring Laccase on Covalently Modified Graphene: Carbon Nanotube Assemblies for the Direct Bio-electrocatalytic Reduction of Oxygen. Chem. Eur. J. 2015, 21, 3198-3201. [CrossRef] [PubMed]

13. Le Goff, A.; Reuillard, B.; Cosnier, S. A Pyrene-Substituted Tris(bipyridine)osmium(II) Complex as a Versatile Redox Probe for Characterizing and Functionalizing Carbon Nanotube- and Graphene-Based Electrodes. Langmuir 2013, 29, 8736-8742. [CrossRef] [PubMed]

14. Fritea, L.; Le Goff, A.; Putaux, J.-L.; Tertis, M.; Cristea, C.; Sandulescu, R.; Cosnier, S. Design of a reduced-graphene-oxide composite electrode from an electropolymerizable graphene aqueous dispersion using a cyclodextrin-pyrrole monomer. Application to dopamine biosensing. Electrochim. Acta 2015, 178, 108-112. [CrossRef]

15. Keeley, G.P.; O'Neill, A.; Holzinger, M.; Cosnier, S.; Coleman, J.N.; Duesberg, G.S. DMF-exfoliated graphene for electrochemical NADH detection. Phys. Chem. Chem. Phys. 2011, 13, 7747-7750. [CrossRef]

16. Lotya, M.; Hernandez, Y.; King, P.J.; Smith, R.J.; Nicolosi, V.; Karlsson, L.S.; Blighe, F.M.; De, S.; Wang, Z.; McGovern, I.T.; et al. Liquid Phase Production of Graphene by Exfoliation of Graphite in Surfactant/Water Solutions. J. Am. Chem. Soc. 2009, 131, 3611-3620. [CrossRef]

17. Smith, R.J.; Lotya, M.; Coleman, J.N. The importance of repulsive potential barriers for the dispersion of graphene using surfactants. New J. Phys. 2010, 12, 125008. [CrossRef]

18. Ramakrishna, T.R.B.; Nalder, T.D.; Yang, W.; Marshall, S.N.; Barrow, C.K. Controlling enzyme function through immobilisation on graphene, graphene derivatives and other two dimensional nanomaterials. J. Mater. Chem. B 2018, 6, 3200-3218. [CrossRef]

19. Rodrigues, R.C.; Ortiz, C.; Berenguer-Murcia, Á.; Torres, R.; Fernández-Lafuente, R. Modifying enzyme activity and selectivity by immobilization. Chem. Soc. Rev. 2013, 42, 6290-6307. [CrossRef]

20. Gravagnuolo, A.M.; Morales-Narváez, E.; Longobardi, S.; da Silva, E.T.; Giardina, P.; Merkoçi, A. In Situ Production of Biofunctionalized Few-Layer Defect-Free Microsheets of Graphene. Adv. Funct. Mater. 2015, 25, 2771-2779. [CrossRef]

21. Zampieri, F.; Wösten, H.A.B.; Scholtmeijer, K. Creating Surface Properties Using a Palette of Hydrophobins. Materials 2010, 3, 4607-4625. [CrossRef]

22. Piscitelli, A.; Pennacchio, A.; Longobardi, S.; Velotta, R.; Giardina, P. Vmh2 hydrophobin as a tool for the development of "self-immobilizing" enzymes for biosensing. Biotechnol. Bioeng. 2017, 114, 46-52. [CrossRef] [PubMed]

23. Sorrentino, I.; Giardina, P.; Piscitelli, A. Development of a biosensing platform based on a laccase-hydrophobin chimera. Appl. Microbiol. Biotechnol. 2019, 103, 3061-3071. [CrossRef] [PubMed]

24. Pezzella, C.; Guarino, L.; Piscitelli, A. How to enjoy laccases. Cell. Mol. Life Sci. 2015, 72, 923-940. [CrossRef] [PubMed]

25. Pezzella, C.; Giacobelli, V.G.; Lettera, V.; Olivieri, G.; Cicatiello, P.; Sannia, G.; Piscitelli, A. A step forward in laccase exploitation: Recombinant production and evaluation of techno-economic feasibility of the process. J. Biotechnol. 2017, 259, 175-181. [CrossRef]

26. Ferrari, A.C.; Meyer, J.C.; Scardaci, V.; Casiraghi, C.; Lazzeri, M.; Mauri, F.; Piscanec, S.; Jiang, D.; Novoselov, K.S.; Roth, S.; et al. Raman Spectrum of Graphene and Graphene Layers. Phys. Rev. Lett. 2006, 97, 187401. [CrossRef]

27. Yaropolov, A.I.; Kharybin, A.N.; Emnéus, J.; Marko-Varga, G.; Gorton, L. Flow-injection analysis of phenols at a graphite electrode modified with co-immobilised laccase and tyrosinase. Anal. Chim. Acta 1995, 308, 137-144. [CrossRef]

28. Freire, R.S.; Thongngamdee, S.; Durán, N.; Wang, J.; Kubota, L.T. Mixed enzyme (laccase/tyrosinase)-based remote electrochemical biosensor for monitoring phenolic compounds. Analyst 2002, 127, 258-261. [CrossRef]

29. Rodríguez-Delgado, M.M.; Alemán-Nava, G.S.; Rodríguez-Delgado, J.M.; Dieck-Assad, G.; Martínez-Chapa, S.O.; Barceló, D.; Parra, R. Laccase-based biosensors for detection of phenolic compounds. Trac. Trends Anal. Chem. 2015, 74, 21-45. [CrossRef]

30. Ferry, Y.; Leech, D. Amperometric Detection of Catecholamine Neurotransmitters Using Electrocatalytic Substrate Recycling at a Laccase Electrode. Electroanalysis 2005, 17, 113-119. [CrossRef] 
31. Palanisamy, S.; Ramaraj, S.K.; Chen, S.-M.; Yang, T.C.K.; Yi-Fan, P.; Chen, T.-W.; Velusamy, V.; Selvam, S. A novel Laccase Biosensor based on Laccase immobilized Graphene-Cellulose Microfiber Composite modified Screen-Printed Carbon Electrode for Sensitive Determination of Catechol. Sci. Rep. 2017, 7, 1-12. [CrossRef]

32. Boujakhrout, A.; Jimenez-Falcao, S.; Martínez-Ruiz, P.; Sánchez, A.; Díez, P.; Pingarrón, J.M.; Villalonga, R. Novel reduced graphene oxide-glycol chitosan nanohybrid for the assembly of an amperometric enzyme biosensor for phenols. Analyst 2016, 141, 4162-4169. [CrossRef] [PubMed]

(C) 2020 by the authors. Licensee MDPI, Basel, Switzerland. This article is an open access article distributed under the terms and conditions of the Creative Commons Attribution (CC BY) license (http://creativecommons.org/licenses/by/4.0/). 